\title{
Effects of Combined Exercise and Low Carbohydrate Ketogenic Diet Interventions on Waist Circumference and Triglycerides in Overweight and Obese Individuals: A Systematic Review and Meta-Analysis
}

\author{
Hyun Suk Lee ${ }^{1(\mathbb{D})}$ and Junga Lee ${ }^{2, *(\mathbb{D})}$ \\ 1 Graduate School of Education, Chung-Ang University, Seoul 06974, Korea; hslee@cau.ac.kr \\ 2 Sports Medicine and Science, Global Campus, Kyung Hee University, Seoul 17104, Korea \\ * Correspondence: jalee@khu.ac.kr; Tel.: +82(31)-201-2738
}

Citation: Lee, H.S.; Lee, J. Effects of Combined Exercise and Low

Carbohydrate Ketogenic Diet Interventions on Waist Circumference and Triglycerides in Overweight and Obese Individuals: A Systematic Review and Meta-Analysis. Int. J. Environ. Res. Public Health 2021, 18, 828. https://doi.org/10.3390/ijerph 18020828

Received: 26 November 2020

Accepted: 11 January 2021

Published: 19 January 2021

Publisher's Note: MDPI stays neutral with regard to jurisdictional claims in published maps and institutional affiliations.

Copyright: (c) 2021 by the authors. Licensee MDPI, Basel, Switzerland. This article is an open access article distributed under the terms and conditions of the Creative Commons Attribution (CC BY) license (https:// creativecommons.org/licenses/by/ $4.0 /)$.

\begin{abstract}
Background: The purpose of this meta-analysis was to investigate the effects of combined exercise and low carbohydrate ketogenic diet interventions (CELCKD) for overweight and obese individuals. (2) Methods: Relevant studies were searched by using the MEDLINE and EMBASE databases up to October 2020. Study Inclusion and Exclusion Criteria: Inclusion criteria were reporting effects of the CELCKD for overweight and obese individuals from randomized controlled trials. Studies that did not match the inclusion criteria were excluded. The methods for CELCKD and outcomes of selected studies were extracted. The effect sizes for interventions that included cardiorespiratory fitness, body composition, fasting glucose, and lipid profiles were calculated by using the standardized mean difference statistic. (3) Results: A total of seven studies and 278 overweight and obese individuals were included. The average intervention of selected studies consisted of moderate to vigorous intensity, 4 times per week for 9.2 weeks. Participating in CELCKD interventions was decreased triglycerides $(d=-0.34, \mathrm{CI} ;-0.68--0.01, p=0.04)$ and waist circumference $(d=-0.74$, $95 \%$ confidence interval $[\mathrm{CI}] ;-1.28--1.20, p=0.01$ ), while cardiovascular fitness, body composition, fasting glucose, total cholesterol, high density lipoprotein (HDL) cholesterol, and low density lipoprotein (LDL) cholesterol were not statistically different after the interventions. No adverse side effects were reported. (4) Conclusions: Participation in interventions by overweight and obese individuals had beneficial effects including decreased waist circumference and triglycerides. Longer term intervention studies with homogenous control groups may be needed.
\end{abstract}

Keywords: exercise; ketogenic diet; obesity; meta-analysis

\section{Introduction}

More than 1.9 billion adults and elders (about 52\% of adults and elders) were overweight and obese in 2016 [1]. Obesity increased threefold from 2016 to 1975 . America had an obesity prevalence of $42.4 \%$ in 2017 2018, which was an increase of 35.0\% in 1999 2000 [2]. Increased populations of overweight and obese individuals were associated with increased chronic diseases including diabetics, metabolic syndrome, cardiovascular diseases, and cancer [3]. Maintaining normal weight has been recommended to help individuals live longer and healthier lives [4].

There were several guidelines that included exercise and a nutritional diet for maintaining normal weight [5]. Recommendations for exercise have included participating in regular moderate intensity aerobic exercise for at least 150 min per week or more than $300 \mathrm{~min}$ per week to achieve long-term weight control; weight lifting at moderate intensity two times per week with 10 to 15 resistance exercise repetitions [6]. Nutritional recommendations have included a $60 \%$ carbohydrate intake, $25 \%$ protein intake, and $15 \%$ fat intake for a balanced nutritional diet and restrictions on calorie intake [7]. Since the last 
couple of decades, previous studies have suggested different diet patterns including low carbohydrate ketogenic diets that limit individuals to less than $130 \mathrm{mg}$ of carbohydrates per day consisting of about $60 \%$ fat intake, $25 \%$ protein intake, and $15 \%$ carbohydrate intake without caloric restrictions [8]. Limiting caloric intake during obesity was the main barrier to participation in weight loss programs. The low carbohydrate ketogenic diet did not have any caloric restrictions, which is attractive to individuals who worry about caloric intake. Additionally, restricted carbohydrate intake triggered insulin release from pancreatic $\beta$ cells during limited stimulations with insulin. The low carbohydrate ketogenic diet increased insulin sensitivity and leptin sensitivity, which reduced feelings of hunger and further helped manage insulin levels. Obese individuals who experience the "yo-yo" effect, a cycle of weight loss and weight regain common in obesity, gain more weight after completing calorically restricted diets [9]. They were looking for easy and effective diets that did not limit caloric intake and improved insulin sensitivity and leptin sensitivity in individuals with metabolic disorders including diabetes and metabolic syndrome. Previous studies of exercise or low carbohydrate ketogenic diets reported reduced weight, improved body composition, and physical outcomes, respectively $[10,11]$. However, several previous studies reported controversial findings and there were several debates about high fat intake [12,13]. Additionally, studies of exercise or diverse diet restrictions showed limited weight loss effects for combined exercise and low carbohydrate ketogenic diet interventions and reported conflicting findings regarding weight loss, body composition, and other physical and psychological factors. While there is no meta-analysis study that included examining the effectiveness of combined exercise and low carbohydrate ketogenic diets for overweight and obese individuals, several review studies reported the effects of those combined interventions. Therefore, the purpose of this meta-analysis was to investigate the effectiveness of combined exercise and a low carbohydrate ketogenic diet for overweight and obese individuals and to provide guidelines for exercise and diet interventions for these individuals.

\section{Materials and Methods}

\subsection{Searching Processes to Identify Eligible Studies}

The guideline for meta-analysis used was the Preferred Reporting Items for Systematic Reviews and Meta-Analyses [14]. Eligible studies were identified by searching the MEDLINE and EMBASE databases for papers published in English up to September 2020. Key words used in this search were "overweight," "obesity," "exercise," "low carbohydrate," "ketogenic diet," "low carbohydrate," AND "high fat," and all possible combinations of those key words. Additionally, lists of citations from review studies were reviewed to identify any missed studies. Two researchers (J. L. and H. L) independently searched for relevant studies. We had further discussions about any disagreements during the selection process. Inclusion criteria for this meta-analysis were reported outcomes of interventions that combined exercise and low carbohydrate ketogenic diets for overweight and obese individuals, studies that investigated a combined exercise intervention and low carbohydrate ketogenic diet, reported participant body mass index (BMI), was a randomized controlled trial, and included overweight and obese individuals (BMI, $>25 \mathrm{~kg} / \mathrm{m}^{2}$ ). Exclusion criteria were review studies, pilot studies, protocol studies, and no report of outcomes due to a combined exercise and low carbohydrate ketogenic diet intervention, no participant BMI, and a protocol that did not include exercise and diet interventions.

\subsection{Statistical Analysis Data Extraction}

Characteristics of the selected studies that included the first author's name, year of publication, country of study, study design, sample size, exercise protocols, low carbohydrate ketogenic protocols including ketone assessments, ketone bodies values to control for diet adherence, and main outcomes, are presented in Table 1. Assessments of quality and risk of bias were rated and are presented in Supplementary Table S1 based on the Cochrane Collaboration's Risk of Bias Tool [15]. The quality and bias risks assessment 
included seven domains: random sequence generation, allocation, concealment, blinding of participants and personnel, blinding of outcomes assessments, complete outcome data, selective reporting, and other biases, and each domain was assessing as low, moderate, or high risk of bias, not as a range of scores.

\subsection{Statistical Analysis}

The standardized mean difference statistic after completing exercise interventions is the difference between treatment and control groups means divided by the pooled standard deviation used to calculate the effect size for each outcome variable. The treatment groups consisted of exercise plus low carbohydrate ketogenic diet and the control groups of exercise plus usual diet. The effect size in this meta-analysis was calculated, when at least two studies reported the same outcomes. Effect size magnitude was interpreted as follows: small effect size was $0.2-0.5$, medium effect size was $0.5-0.8$, and a large effect size was $>0.8$. Heterogeneity among the effect sizes was determined by the Q statistic. When $\mathrm{I}^{2}$ was $\leq 50 \%$, heterogeneity was absent using a fixed-effects model. When $\mathrm{I}^{2}$ was $>50 \%$, heterogeneity did exist using used a random effects model. Comprehensive Meta-Analysis Version 1.25 software (Biostatic Inc, Englewood, NJ, USA) was used to complete all analyses. 
Table 1. Basic characteristics of selected studies.

\begin{tabular}{|c|c|c|c|c|}
\hline First Author (Year), Country & Design, Number of Participants Per Group, Age & Exercise Intervention & Ketogenic Diet & Outcomes \\
\hline Gregory (2017), USA & $\begin{array}{l}\text { RCT: exercise }+ \text { ketogenic diet }(n=12) \text { vs. control } \\
\qquad(n=15), 34.58 \pm 9.26 \text { years old }\end{array}$ & 6 weeks, 4 CrossFit workouts/week & $\begin{array}{l}6 \text { weeks, restricting carbohydrate intake } \\
\text { to no more than } 50 \mathrm{~g} / \text { week }(<10 \% \text { of } \\
\text { energy), given a detailed guide on } \\
\text { acceptable low-carbohydrate, fat, protein } \\
\text { rich foods, keeping dietary food intake } \\
\text { records, urinary ketone: ketosis }> \\
15 \mathrm{mg} / \mathrm{dl}\end{array}$ & $\begin{array}{l}\text { Body composition (weight, body mass } \\
\text { index (BMI), body fat, fat mass, lean } \\
\text { body mass), performance (vertical jump, } \\
\text { standing long jump, total } \\
\text { performance time) }\end{array}$ \\
\hline Gyorkos (2019), USA & $\begin{array}{l}\text { RCT: exercise }+ \text { carbohydrate-restricted } \\
\text { Paleolithic-base diet }(n=12) \text {, vs. control }(n=12) \\
40.9 \pm 20.2 \text { years old })\end{array}$ & $\begin{array}{l}4 \text { weeks, high intensity interval training } \\
10 \times 60 \mathrm{~s} \text { cycling intervals and } 60 \mathrm{~s} \text { of } \\
\text { recovery, } 3 \text { days } / \text { week }\end{array}$ & $\begin{array}{l}4 \text { weeks, carbohydrate-restricted } \\
\text { Paleolithic based diet, protein }(25 \%) \text {, fat } \\
(60 \%) \text {, carbohydrate }(15 \%, 50 \mathrm{~g} / \mathrm{d}) \text {, no } \\
\text { restricted calories, blood ketone } \\
\text { ß-hydroxybutyrate ketone } \\
\text { level: } 0.53 \pm 0.29 \mathrm{mmol} / \mathrm{L}\end{array}$ & $\begin{array}{l}\text { Dietary intake (energy, protein, } \\
\text { carbohydrate, total, fat, saturated fat, } \\
\text { monounsaturated fat, polyunsaturated } \\
\text { fat, alcohol, cholesterol), brain derived } \\
\text { neurotrophic factor (BDNF), cognitive } \\
\text { symptoms and function, cognitive speed } \\
\text { and flexibility, cognitive flexibility }\end{array}$ \\
\hline Sun (2019), China & $\begin{array}{l}\text { RCT: high intensity interval training + low } \\
\text { carbohydrate (HIIT, } n=18,20.8 \pm 2.7 \text { years old), } \\
\text { moderate intensity continuous training + low } \\
\text { carbohydrate (MICT, } n=13,21.5 \pm 3.1 \text { years old), } \\
\text { control + low carbohydrate (LC-CON, } n=15 \\
20.9 \pm 3.7 \text { years old), vs. control }(n=15 \\
21.6 \pm 3.9 \text { years old })\end{array}$ & $\begin{array}{l}4 \text { weeks, high intensity interval training } \\
(10 \times 6 \mathrm{~s} \text { cycling sprints and } 9 \mathrm{~s} \text { recovery, } \\
\text { total } 2.5 \mathrm{~min} / \text { session), moderate } \\
\text { intensity continuous training ( } 30 \mathrm{~min} \\
\text { cycling, } 5 \text { min for warm-up, } 50 \% \text { of } \\
\mathrm{VO}_{2 \text { peak }} \text { for } 10 \text { min, } 60 \% \text { of } \mathrm{VO}_{2 \text { peask }} \text { for } \\
10 \text { min, } 5 \text { min for recovery), } \\
5 \text { days/week, pedometers, logbook }\end{array}$ & $\begin{array}{c}4 \text { weeks, carbohydrate }(10 \%, \sim 50 \mathrm{~g} / \mathrm{d}) \text {, } \\
\text { fat }(65 \%), \text { protein }(\sim 25 \%), \text { urinary ketone } \\
\text { was detected: } 97.6 \pm 4.5 \% \text { in LC-CON, } \\
96.2 \pm 8.3 \% \text { in HIIT, and } 96.9 \pm 6.0 \% \text { in } \\
\text { the MICT, 3-day food records }\end{array}$ & $\begin{array}{l}\text { Weight, BMI, waist circumference, } \\
\text { waist-to-hip ratio, } \mathrm{VO}_{2 \text { peak, }} \text { fasting } \\
\text { glucose, total cholesterol, HDL, LDL, } \\
\text { triglycerides, }\end{array}$ \\
\hline $\begin{array}{l}\text { Perissiou (2020), United } \\
\text { Kingdom }\end{array}$ & $\begin{array}{l}\text { RCT: exercise }+ \text { low carbohydrate }(n=33), \mathrm{vs} \text {. } \\
\text { control (exercise }+ \text { standard diet, } n=31) \\
35.3 \pm 9 \text { years old }\end{array}$ & $\begin{array}{l}8 \text { weeks, a combination of aerobic and } \\
\text { resistance exercise, } 60 \% \sim 70 \% \text { heart rate } \\
\text { (HR) peak and } 85 \% \sim 95 \% \text { HRpeak, } \\
\text { supervised (1st } \sim 2 \text { nd week) and } \\
\text { unsupervised (3rd } \sim 8 \text { th week) }\end{array}$ & $\begin{array}{c}8 \text { weeks, carbohydrate }(>50 \mathrm{~g} / \mathrm{d}) \\
\text { B-hydroxybutyrate ketone level: } \\
>0.3 \mathrm{mmol} / \mathrm{L}\end{array}$ & $\begin{array}{l}\text { Cardiorespiratory fitness }\left(\mathrm{VO}_{2 \text { peak }}\right), \\
\text { Total cholesterol, HDL, } \mathrm{LDL}, \\
\text { triglycerides, fasting glucose, CRP, } \\
\text { adiponectin, blood pressure, body } \\
\text { weight, total body fat, visceral adipose } \\
\text { tissue, lean muscle, mass, fat mass index, } \\
\text { total bone mineral density }\end{array}$ \\
\hline Freedland (2019), USA & $\begin{array}{l}\text { RCT: exercise }+ \text { low carbohydrate diet }(n=11) \text {, vs. } \\
\quad \text { control }(n=18) \text {, average } 66 \text { years old }\end{array}$ & 24 weeks, $\geq 30 \mathrm{~min} / \mathrm{da}, \geq 5$ days/week & $\begin{array}{l}6 \text { months, low carbohydrate }(\leq 20 \mathrm{~g} / \text { day }) \\
\text { until urinary ketone bodies detected }\end{array}$ & $\begin{array}{l}\text { Carbohydrates, fat, protein, calories, } \\
\text { weight, BMI, HOMA, glucose, insulin, } \\
\text { HbA1c, cholesterol, LDL, HDL, } \\
\text { non-HDL, triglycerides, prostate-specific } \\
\text { antigen, HsCRP }\end{array}$ \\
\hline
\end{tabular}


Table 1. Cont.

\begin{tabular}{|c|c|c|c|c|}
\hline First Author (Year), Country & Design, Number of Participants Per Group, Age & Exercise Intervention & Ketogenic Diet & Outcomes \\
\hline LaFountain (2019), USA & $\begin{array}{c}\text { Prospective intervention study: exercise }+ \\
\text { ketogenic diet }(n=17), 27.4 \pm 6.8 \text { years old vs. } \\
\text { exercise + mixed diet }(n=17), 24.6 \pm 9.0 \text { years old }\end{array}$ & $\begin{array}{l}12 \text { weeks, progressive resistance training } \\
\text { ( } 2 \text { days } / \text { week, } \sim 60 \text { min/session, } 3 \text { sets of } \\
12 \text { reps at } 60 \%-1 \text { repetition maximum } \\
\text { (RM) to } 4 \text { sets of } 4 \text { reps at } 95 \%-1 \mathrm{RM} \text { ), and } \\
\text { cardiopulmonary fitness (running, } \\
\text { body-weight circuit training, } 30 \mathrm{~min} \text { ) }\end{array}$ & $\begin{array}{c}\text { Limited carbohydrate }(25 \mathrm{~g} / \mathrm{d}) \text { and } \\
\text { protein }(90 \mathrm{~g} / \mathrm{d}) \text {, sodium }(4 \sim 5 \mathrm{~g} / \mathrm{d}), \\
\text { alcohol }(\geq 2 \text { drinks } / \text { day }), \text { a ketone } \\
\text { monitor: } \beta \text {-hydroxybutyrate ketone } \\
\text { level: } 1.2 \pm 0.3 \mathrm{mM}\end{array}$ & $\begin{array}{l}\text { Basal metabolic rate (RMR), relative } \\
\text { RMR, respiratory exchange ratio, } \% \\
\text { carbohydrate, } \% \text { fat, anaerobic } \\
\text { performance (1RM strength, counter } \\
\text { movement, } 10 \text { s sprint intervals), resting } \\
\text { metabolic rate }\end{array}$ \\
\hline Jabekk (2010), Norway & $\begin{array}{l}\text { RCT: exercise }+ \text { ketogenic diet }(n=8) \text {, vs. exercise + } \\
\quad \text { regular diet }(n=8) \text {, between } 20 \sim 40 \text { years }\end{array}$ & $\begin{array}{c}10 \text { weeks, resistance exercise (12 RM, } \\
\text { supine leg press, seated leg extension, } \\
\text { seated leg curl, seated chest press, seated } \\
\text { rowing, seated shoulder press, seated up } \\
\text { down, standing biceps curl) }\end{array}$ & $\begin{array}{l}10 \text { weeks, restricted carbohydrates } \\
\text { intake until urinary ketone bodies } \\
\text { detected, urine reagent strips }\end{array}$ & $\begin{array}{l}\text { Body weight, lean body mass, fasting } \\
\text { blood lipids, glucose, dietary nutrition }\end{array}$ \\
\hline
\end{tabular}




\section{Results}

The selection process for this meta-analysis is presented in Figure 1. A total of 3045 studies from the initial screening were found. The titles and abstracts from the initial screening were reviewed and 3007 studies, which were review studies, pilot studies, or not related to this meta-analysis, were excluded. Full texts of the remaining 38 studies were reviewed to determine eligibility, and finally, seven studies were selected [16-21]. A total of 255 overweight and obese individuals were included in this meta-analysis. Four studies were conducted in America, two studies in Europe, and one study in Asia. A control group of exercise and usual dietary intake was included. Quality and risk of bias assessments for selected studies were conducted using the Cochrane Collaboration's Risk of Bias Tool, without the total scores or cutoff scores for quality and risk of bias, which maybe have produced unclear results with respect to establishing the quality and risk of bias (Supplementary Table S1). We did not find a high risk of bias when assessing the quality of risk bias. Adverse side effects for the combined exercise and low carbohydrate ketogenic diet interventions were not reported.

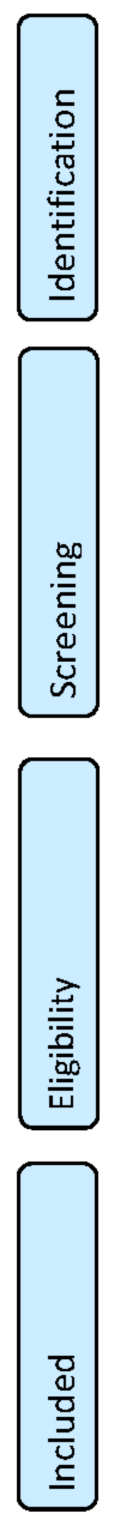

Records identified through database searching ( $n=3045$ ) sources $(n=0)$

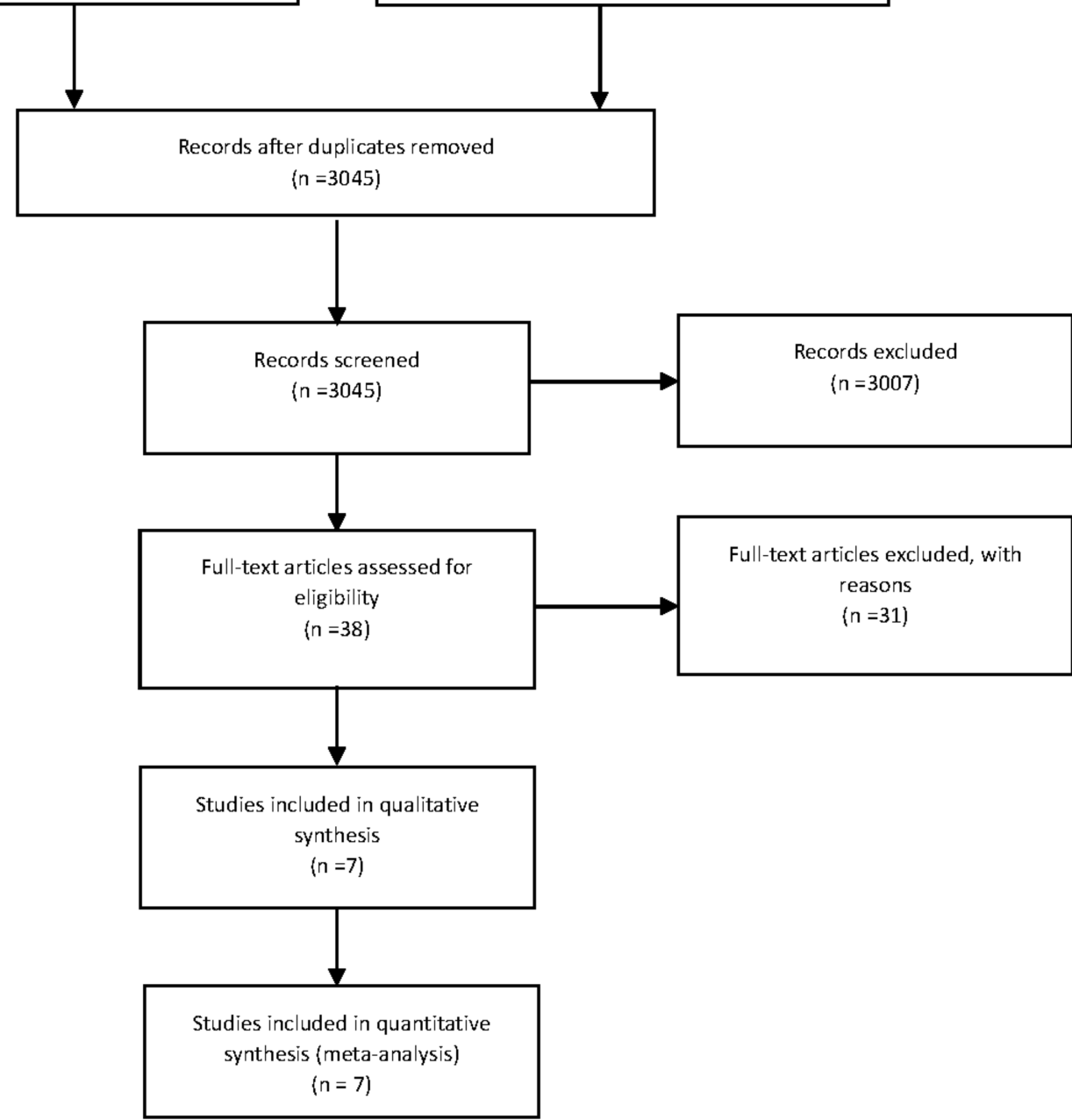

Figure 1. Selection process for the systematic review and meta-analysis. 


\subsection{Exercise and Ketogenic Diet Interventions}

Average periods of exercise and ketogenic diet intervention ranged from 4 to 24 weeks (9.2 weeks). Three studies included high intensity interval training, two studies used moderate intensity aerobic exercise, three studies used resistance exercise, and one study used CrossFit exercise. Average frequency and duration of exercise was 4 times per week for one hour. Ketogenic diet components averaged $65 \%$ fat, $25 \%$ protein, and $10 \%$ carbohydrates and did not restrict calorie intake. The ketone levels of participants or their daily food diaries were recorded and used to assess the status of the ketogenic diet.

\subsection{Body Compositions}

Combined exercise and ketogenic diet groups were included in 6 trials, body mass index (BMI) was included in 5 trials, \% body fat mass in 3 trials, lean body mass in 2 trials, waist circumference in 2 trials. There was a statistically significant decrease in waist circumference compared to the control group $(d=-0.74,95 \%$ confidence interval [CI]; $-1.24-0.20, p=0.01])$. Body mass index (BMI) $(d=-0.33,95 \%$ confidence interval $[\mathrm{CI}] ;-0.68-0.02, p=0.67]), \%$ body fat mass $(d=-0.06,95 \%$ confidence interval $[\mathrm{CI}] ;-0.44-0.32, p=0.78])$, and lean body mass $(d=-0.77,95 \%$ confidence interval $[\mathrm{CI}]$; $-2.45-0.90, p=0.37]$ ) showed not statistically significant effect sizes post-intervention compared to control groups.

\subsection{Cardiorespiratory Fitness $\left(V O_{2 p e a k}\right)$}

Subjects who participated in 4 trials of combined exercise and ketogenic diet did not have a statistically significant increase in cardiorespiratory fitness $(d=0.18,95 \%$ confidence interval [CI]; $-0.17-0.41, p=0.43]$ ) compared to the control groups (Figure 2).

\subsection{Fasting Glucose and Lipid Profiles}

Combined exercise and ketogenic diet groups in 4 trials had statistically significantly decreased triglycerides $(d=-0.34,95 \% \mathrm{CI} ;-0.68--0.01, p=0.04)$ compared to the control groups. Fasting glucose $(d=-0.01,95 \%$ confidence interval [CI]; $-0.40-0.39, p=0.98])$, total cholesterol $(d=0.20,95 \%$ confidence interval $[C I] ;-0.14-0.53, p=0.25])$, low-density lipoprotein (LDL) $(d=0.46,95 \%$ confidence interval [CI]; $-0.02-0.95, p=0.65])$, and highdensity lipoprotein (HDL) $(d=0.18,95 \%$ confidence interval [CI]; $-0.16-0.51, p=0.31])$ were not statistically significant post-intervention compared to control groups. 


\begin{tabular}{|c|c|c|c|c|c|c|c|c|c|c|c|c|}
\hline \multicolumn{9}{|c|}{ Waist circumference } & \multicolumn{4}{|c|}{ Test for heterogeneity: $\left(\mathrm{Q}=2.21, \mathrm{P}=0.82, \mathrm{I}^{2}=0.00 \%\right)$} \\
\hline $\begin{array}{l}\text { Study } \\
\text { name }\end{array}$ & $\begin{array}{l}\text { Exercis } \\
\text { e mean }\end{array}$ & $\begin{array}{l}\text { Exercise } \\
\text { SD }\end{array}$ & $\begin{array}{l}\text { Exercise } \\
\mathrm{N}\end{array}$ & $\begin{array}{l}\text { Control } \\
\text { mean }\end{array}$ & $\begin{array}{l}\text { Control } \\
\text { SD }\end{array}$ & $\begin{array}{l}\text { Control } \\
\mathrm{N}\end{array}$ & $\begin{array}{l}\text { Weig } \\
\text { ht } \\
\text { to }\end{array}$ & WDN[95\%Cl] & \multicolumn{4}{|c|}{ Stdatlinnears ad $99 \%$ CI } \\
\hline $\begin{array}{l}\text { Sun, } 2019 \\
\text { high } \\
\text { exercise }\end{array}$ & 75.70 & 9.50 & 15 & 78.50 & 9.20 & 15 & 56.34 & $-0.30[-1.02,0.42]$ & & 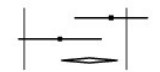 & & \\
\hline $\begin{array}{l}\text { Sun, } 2019 \\
\text { moderate } \\
\text { exercise }\end{array}$ & 71.20 & 4.80 & 13 & 78.50 & 6.20 & 15 & 43.66 & $-1.30[-2.12,-0.49]$ & 400 & $\begin{array}{ll}-200 & 000 \\
& \end{array}$ & $\begin{array}{l}200 \\
\text { Fovours Conra }\end{array}$ & 40 \\
\hline & & & & & & & & $-0.74[-1.28,-0.20]$ & & & & \\
\hline
\end{tabular}

(a)

\begin{tabular}{|c|c|c|c|c|c|c|c|c|c|c|c|c|}
\hline \multicolumn{9}{|c|}{ Weight } & \multicolumn{4}{|c|}{ Test for heterogeneity: $\left(Q=2.21, P=0.82, I^{2}=0.00 \%\right)$} \\
\hline $\begin{array}{l}\text { Study } \\
\text { name }\end{array}$ & $\begin{array}{l}\text { Exercis } \\
\text { e mean }\end{array}$ & $\begin{array}{l}\text { Exercise } \\
\text { SD }\end{array}$ & $\begin{array}{l}\text { Exercise } \\
\mathrm{N}\end{array}$ & $\begin{array}{l}\text { Control } \\
\text { mean }\end{array}$ & $\begin{array}{l}\text { Control } \\
\text { SD }\end{array}$ & $\begin{array}{l}\text { Control } \\
\mathrm{N}\end{array}$ & $\begin{array}{l}\text { Weight } \\
(\%)\end{array}$ & WDN $[95 \% \mathrm{Cl}]$ & & 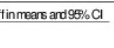 & & \\
\hline $\begin{array}{l}\text { Gregory, } \\
2017\end{array}$ & 73.18 & 12.51 & 18 & 70.30 & 12.65 & 15 & 13.99 & $0.04[-0.72,0.80]$ & & $\square$ & & \\
\hline $\begin{array}{l}\text { Freedland, } \\
2019\end{array}$ & 88.50 & 11.60 & 11 & 91.3 & 14.10 & 18 & 14.26 & $-0.21[-0.96,0.54]$ & & $\div$ & & \\
\hline $\begin{array}{l}\text { Jabekk, } \\
2010\end{array}$ & 90.00 & 8.80 & 8 & 87.00 & 7.90 & 8 & 8.26 & $0.36[-0.63,1.35]$ & & $\bar{a}_{a \infty}$ & 200 & $\left.\right|_{4 \infty} ^{1}$ \\
\hline $\begin{array}{l}\text { Perrisiou, } \\
2020\end{array}$ & 83.36 & 16.80 & 31 & 82.90 & 12.89 & 33 & 33.55 & $0.03[-0.46,0.52]$ & & & Favours Contra & \\
\hline $\begin{array}{l}\text { Sun, } 2010 \\
\text { high }\end{array}$ & 65.00 & 9.70 & 15 & 66.10 & 10.8 & 15 & 15.72 & $-0.11[-0.82,0.61]$ & & & & \\
\hline $\begin{array}{l}\text { Sun, } 2010 \\
\text { moderate } \\
\text { exercise }\end{array}$ & 61.90 & 6.00 & 13 & 66.10 & 10.8 & 15 & 14.22 & $-0.47-0.35,0.22]$ & & & & \\
\hline & & & & & & & & $-0.07-0.35,0.22]$ & & & & \\
\hline
\end{tabular}

(b)

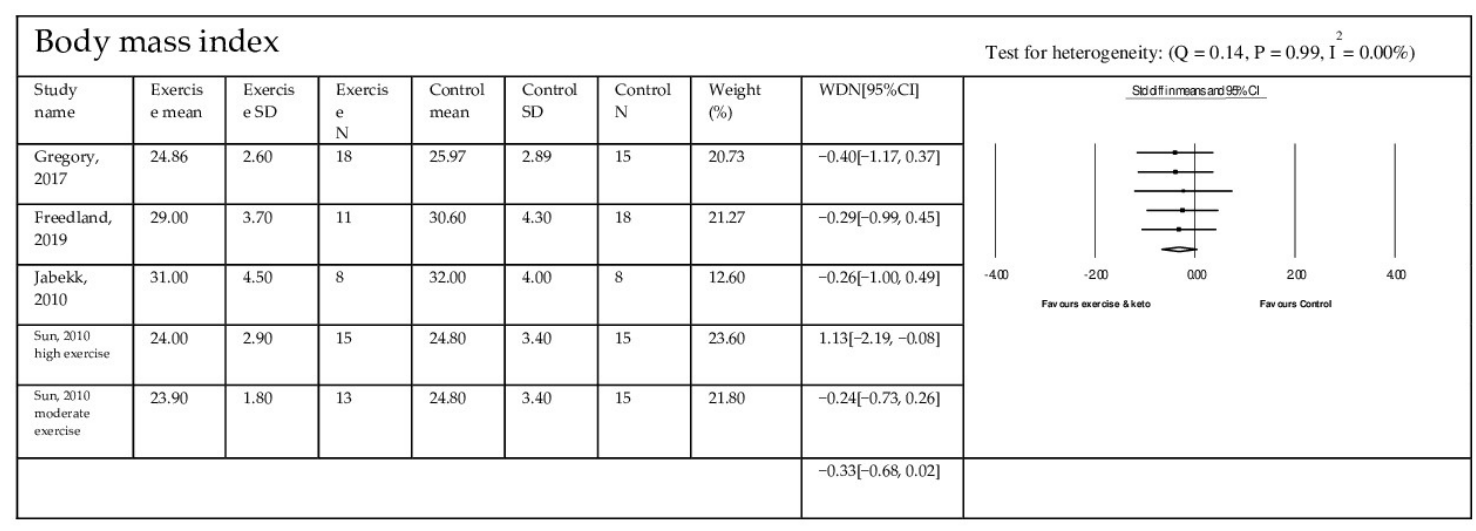

(c)

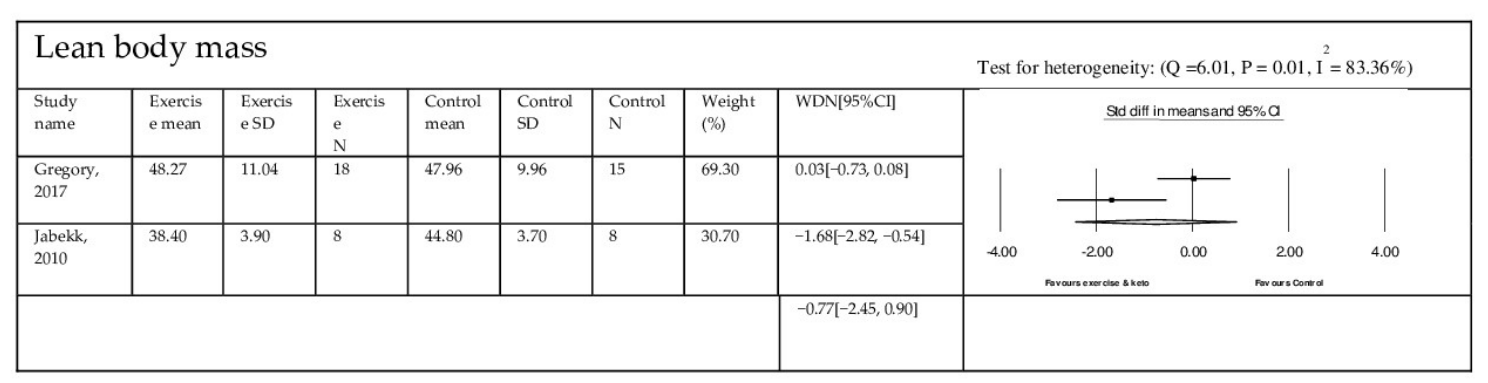

(d)

Figure 2. Cont. 


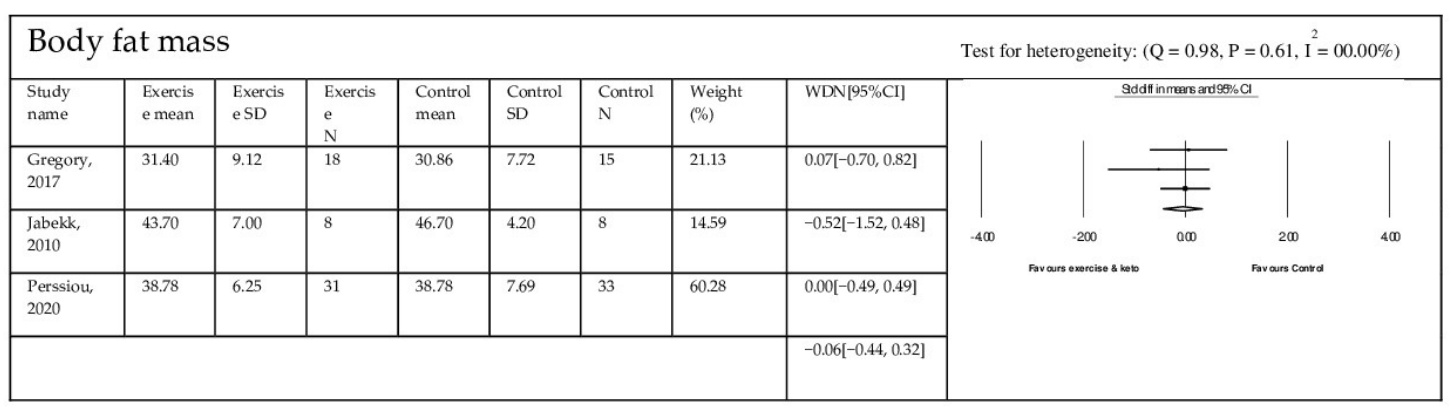

(e)

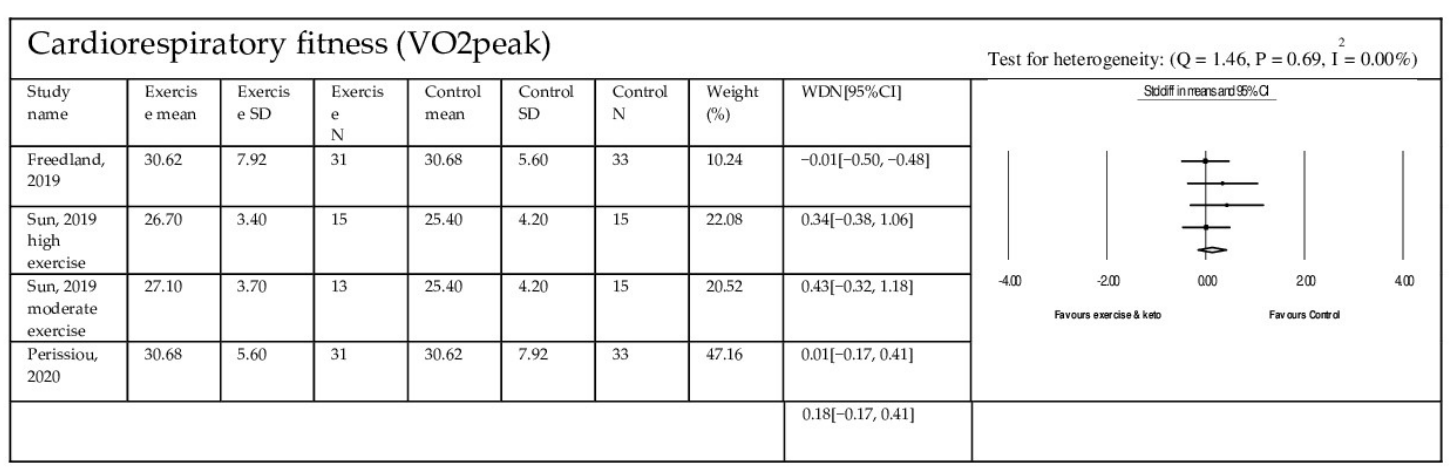

(f)

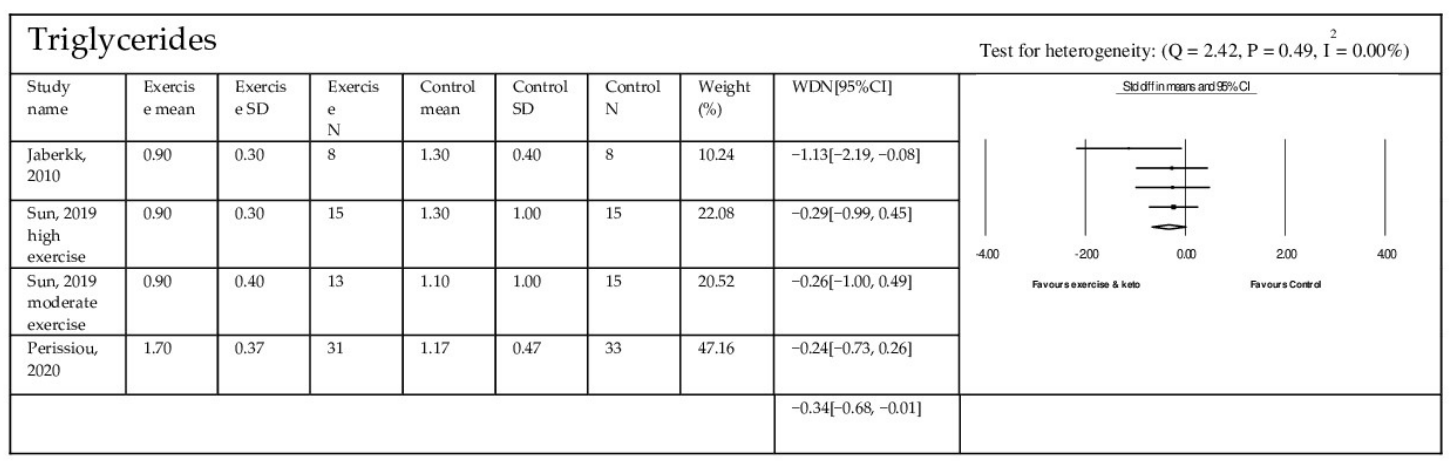

(g)

\begin{tabular}{|c|c|c|c|c|c|c|c|c|c|c|c|c|}
\hline \multicolumn{9}{|c|}{ Total cholesterol } & \multicolumn{4}{|c|}{ Test for heterogeneity: $\left(Q=2.54, P=0.47, I^{2}=00.00 \%\right)$} \\
\hline $\begin{array}{l}\text { Study } \\
\text { name }\end{array}$ & $\begin{array}{l}\text { Exercis } \\
\text { e mean }\end{array}$ & $\begin{array}{l}\text { Exercis } \\
\text { eSD }\end{array}$ & $\begin{array}{l}\text { Exercis } \\
e_{\mathrm{N}} \\
\mathrm{N}\end{array}$ & $\begin{array}{l}\text { Control } \\
\text { mean }\end{array}$ & $\begin{array}{l}\text { Control } \\
\text { SD }\end{array}$ & $\begin{array}{l}\text { Control } \\
\mathrm{N}\end{array}$ & Weight $(\%)$ & WDN[95\%Cl] & \multirow{5}{*}{-40} & \multirow{5}{*}{$\overline{E_{0}}$} & \multirow{5}{*}{$\begin{array}{c}1 \\
200 \\
\text { Fmours Control }\end{array}$} & \multirow{5}{*}{$\left.\right|_{400} ^{1}$} \\
\hline $\begin{array}{l}\text { Jebekk, } \\
2010\end{array}$ & 5.70 & 1.20 & 8 & 4.80 & 0.70 & 8 & 10.66 & $0.92[-0.11,1.95]$ & & & & \\
\hline $\begin{array}{l}\text { Sun, } 2019 \\
\text { high } \\
\text { exercise }\end{array}$ & 4.7 & 0.60 & 15 & 4.70 & 0.30 & 15 & 22.08 & $0.00[-0.72,0.72]$ & & & & \\
\hline $\begin{array}{l}\text { Sun, } 2019 \\
\text { moderate } \\
\text { exercise }\end{array}$ & 4.8 & 0.30 & 13 & 4.70 & 0.30 & 15 & 20.23 & $0.33[-0.41,1.08]$ & & & & \\
\hline $\begin{array}{l}\text { Perrissiou, } \\
2020\end{array}$ & 4.62 & 0.92 & 31 & 4.56 & 0.75 & 33 & 47.03 & $0.07[-0.42,0.52]$ & & & & \\
\hline & & & & & & & & $0.20[-0.14,0.53]$ & & & & \\
\hline
\end{tabular}

(h)

Figure 2. Cont. 


\begin{tabular}{|c|c|c|c|c|c|c|c|c|c|c|c|}
\hline \multicolumn{9}{|c|}{ LDL cholesterol } & \multicolumn{3}{|c|}{ Test for heterogeneity: $\left(\mathrm{Q}=5.43, \mathrm{P}=0.14, \mathrm{I}^{2}=44.70 \%\right)$} \\
\hline $\begin{array}{l}\text { Study } \\
\text { name }\end{array}$ & $\begin{array}{l}\text { Exercis } \\
\text { e mean }\end{array}$ & $\begin{array}{l}\text { Exercis } \\
\text { eSD }\end{array}$ & $\begin{array}{l}\text { Exercis } \\
e\end{array}$ & $\begin{array}{l}\text { Control } \\
\text { mean }\end{array}$ & $\begin{array}{l}\text { Control } \\
\text { SD }\end{array}$ & $\begin{array}{l}\text { Control } \\
N\end{array}$ & $\begin{array}{l}\text { Weight } \\
(\%)\end{array}$ & WDN[95\%Cl] & \multirow{5}{*}{ 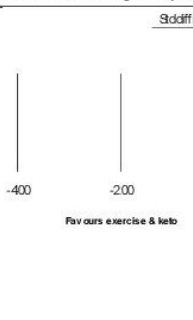 } & \multirow{5}{*}{$\underset{0}{\rightleftarrows}$} & \multirow{5}{*}{40} \\
\hline $\begin{array}{l}\text { Jebekk, } \\
2010\end{array}$ & 5.70 & 1.20 & 8 & 4.80 & 0.70 & 8 & 16.00 & $0.90[-0.13,1.93]$ & & & \\
\hline $\begin{array}{l}\text { Sun, } 2019 \\
\text { high } \\
\text { exercise }\end{array}$ & 4.7 & 0.60 & 15 & 4.70 & 0.30 & 15 & 2420 & $0.85[0.10,1.60]$ & & & \\
\hline $\begin{array}{l}\text { Sun, } 2019 \\
\text { moderate } \\
\text { exercise }\end{array}$ & 4.8 & 0.30 & 13 & 4.70 & 0.30 & 15 & 23.89 & $0.53\{-0.23,1.29]$ & & & \\
\hline $\begin{array}{l}\text { Perrissiou, } \\
2020\end{array}$ & 4.62 & 0.92 & 31 & 4.56 & 0.75 & 33 & 35.91 & $-0.04[-0.53,0.45]$ & & & \\
\hline & & & & & & & & $0.46[-0.02,0.95]$ & & & \\
\hline
\end{tabular}

(i)

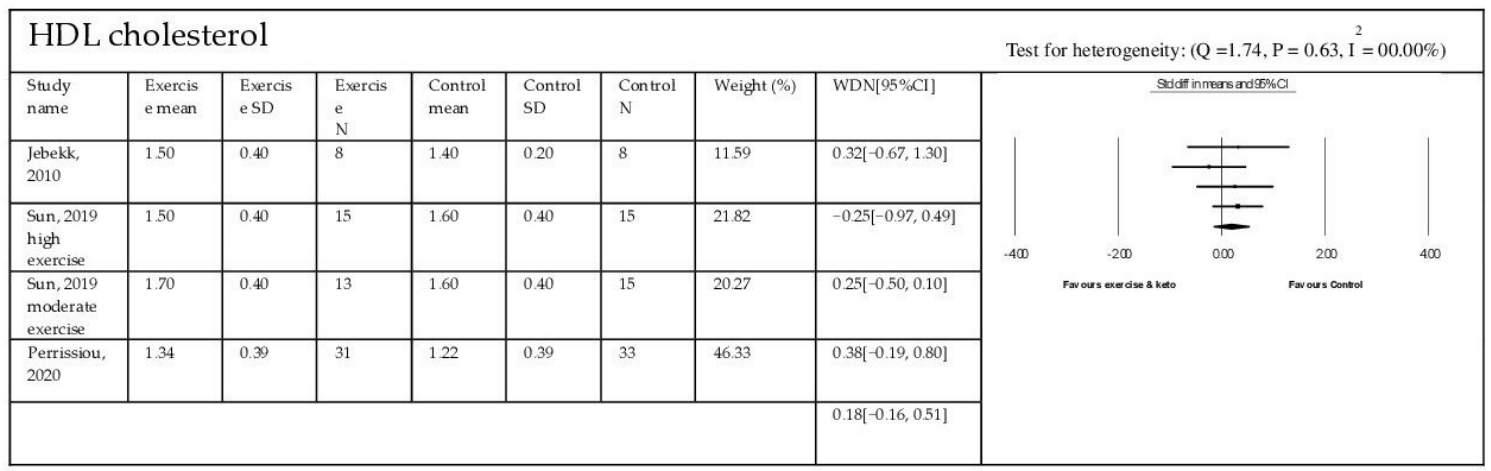

(j)

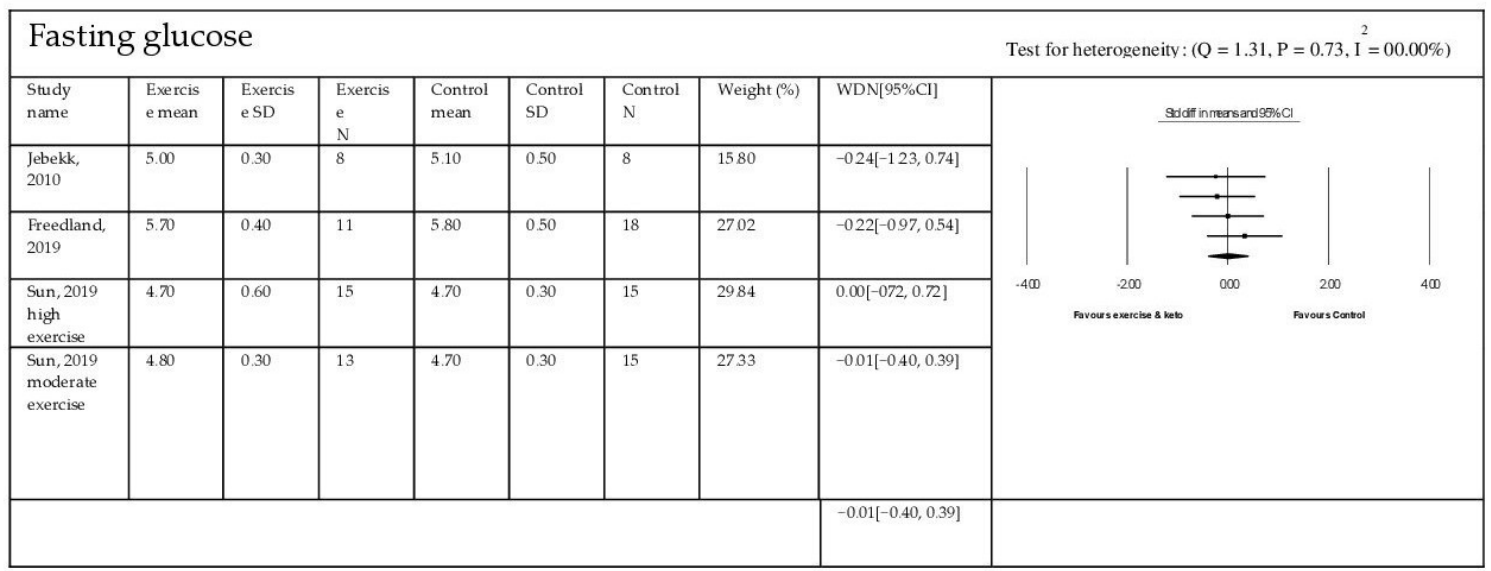

(k)

Figure 2. Effect sizes of exercise and ketogenic diet intervention for overweight and obese individual: (a) Effects of combined exercise and low carbohydrate ketogenic diet interventions on waist circumference, (b) Effects of combined exercise and low carbohydrate ketogenic diet interventions on weight, (c) Effects of combined exercise and low carbohydrate ketogenic diet interventions on body mass index, (d) Effects of combined exercise and low carbohydrate ketogenic diet interventions on lean body mass, (e) Effects of combined exercise and low carbohydrate ketogenic diet interventions on body fat mass, (f) Effects of combined exercise and low carbohydrate ketogenic diet interventions on Cardiorespiratory fitness $\left(\mathrm{VO}_{2 p e a k}\right),(\mathrm{g})$ Effects of combined exercise and low carbohydrate ketogenic diet interventions on Triglycerides, (h) Effects of combined exercise and low carbohydrate ketogenic diet interventions on total cholesterol, (i) Effects of combined exercise and low carbohydrate ketogenic diet interventions on LDL cholesterol, (j) Effects of combined exercise and low carbohydrate ketogenic diet interventions on HDL cholesterol, (k) Effects of combined exercise and low carbohydrate ketogenic diet interventions on fasting glucose. 


\section{Discussion}

Overweight and obese individuals in combined exercise and low carbohydrate ketogenic diet interventions had beneficial effects post-intervention that included decreased waist circumference and triglycerides compared to control groups. Body composition metrics, fasting glucose, and lipid profiles were not statistically different post-intervention compared to control groups. While more studies may be needed, the effects of combined exercise and ketogenic diet interventions can help to improve body composition and triglycerides. The reduction in waist circumference and triglycerides without body mass and body composition changes was clinically meaningful because waist circumference and triglycerides are inversely associated with risks of chronic disease [22,23].

Participating in combined exercise and low carbohydrate ketogenic diet interventions helped reduce waist circumference in overweight and obese individuals. No meta-analysis of these effects has been reported previously, and several individual previous studies were conflicting $[17,18]$. The current meta-analysis found decreased waist circumference in the combined exercise and low carbohydrate ketogenic diet intervention groups compared to control groups. Nevertheless, more studies are needed to confirm these results due to the limited sample size in this meta-analysis study. Decreased waist circumference was meaningful because it has an inverse association with chronic diseases [22,24]. Compared to control groups in the current meta-analysis, body composition metrics including weight, BMI, \% body fat, and lean body mass were not statistically different post-intervention. These results may be clearer when more studies are conducted and homogeneous control groups are included, no treatments included exercise interventions with standard diet as control groups. Additionally, other measurements of body composition including visceral fat and other ectopic fats using computed tomography (CT), magnetic resonance imaging (MRI), volume-localized 1H-magnetic resonance spectroscopy (MRS), and dual-energy X-ray absorptiometry (DAX) are needed to assess body composition metrics that provide more specific results.

Cardiorespiratory fitness was increased after combined exercise and low carbohydrate ketogenic diet interventions when compared to control groups, but this effect was not statistically significant. One reason for this lack of statistically significant effect is because participants in both the intervention groups and control groups included exercise and the only difference between the groups was whether the diet was a low carbohydrate ketogenic diet or a usual diet. Additional studies are needed to make proper conclusions about the intervention effects of exercise plus a low carbohydrate ketogenic diet as the experimental group and low carbohydrate ketogenic diet only as the control group. A recent meta-analysis reported that higher cardiorespiratory fitness among overweight and obese individuals was related to a lower incidence of metabolic syndrome compared to individuals with lower cardiorespiratory fitness [25]. Improving cardiorespiratory fitness is important for overweight and obese individuals to reduce the incidence of chronic diseases which may be affected by exercise rather than diet.

Overweight and obese individuals in the combined exercise and low carbohydrate ketogenic diet intervention groups showed the favorable effect of reduced triglycerides. Current findings for decreased triglycerides are supported by several previous studies $[20,26]$. An aerobic exercise training intervention in obese participants showed decreased hepatic and visceral lipids including triglycerides without weight loss [27]. Additionally, obese individuals who had a ketogenic diet showed decreased circulating triglycerides independent of significant weight loss [28]. The combined effects of exercise and ketogenic diet showed reduced triglycerides in overweight and obese individuals. Current findings did not show statistically significant changes in other lipid profiles, including total cholesterol, LDL, and HDL. More studies are needed since other findings from previous studies were controversial $[17,19,20]$. While our findings regarding lipid profiles did not show statistically significant changes, a previous study reported a significantly decreased lipid profile. The findings of other previous studies coincided with our findings. While there are several debates regarding high fat intake that may be associated with increased risks of 
cardiovascular diseases [29], the selected studies in the current meta-analysis did not report any adverse side effects. The average length of the combined intervention with exercise and low carbohydrate ketogenic diet in our meta-analysis was 9.2 weeks. Longer-term studies of the interventions including exercise and high fat diets need to occur to confirm side effects.

The exercise interventions in our meta-analysis were moderate to vigorous aerobic and resistance exercise, at an average of 4 times per week, and more than 60 min per day for 9.2 weeks. The average length of the exercise interventions agreed with the recommended guidelines of the American College of Sports Medicine for obesity. These guidelines recommend moderate to vigorous aerobic activity starting with a minimum of 30 min per day, increasing to $60 \mathrm{~min}$ per day, and including resistance exercise, flexibility exercises, and activities of daily living. In addition, the low carbohydrate ketogenic diet consisted of low carbohydrate intake ( $<50 \mathrm{~g} /$ day, $<10 \%$ of energy), high fat intake ( $>65 \%$ of energy), and protein intake ( $>25 \%$ of energy). The length of the low carbohydrate ketogenic diet interventions in the selected studies ranged from 4 to 24 weeks. An initial period of 2 weeks in the low carbohydrate ketogenic diet allowed the subjects to adapt to the new diet and reveal several side effects such as dizziness, fatigue, and stomach pain. The severity of these side effects was low enough to not warrant extensive study in future research.

There were several strengths and limitations to this current meta-analysis. This meta-analysis is the first meta-analysis to report the effects of combining exercise and low carbohydrate ketogenic diet in overweight and obese individuals from randomized controlled trials by analyzing effect sizes of the outcomes of the interventions. In addition, we are presenting in detail effective exercise programs from the selected studies as exercise guides for overweight and obese individuals. There were several limitations in this metaanalysis. First, the number of selected studies was too small to clarify current findings, even though all relevant studies were searched. Second, participants in the control groups in the selected studies underwent exercise interventions with usual diet. The exercise interventions in the control groups were not systematically controlled or designed in a way that let the subjects participate in their usual physical activity and exercise or the same exercise interventions as the experimental groups. This participant sample was small such that our results may have underestimated the effects of the combined interventions. Third, a total of 278 participants were included in this meta-analysis, and many more participants are needed to allow for a wide generalization of our findings. Only one study was conducted in Asia, which shows the need for more diverse cohorts. Lastly, the length of the exercise and low carbohydrate ketogenic interventions was an average of 9.2 weeks except for one study, which lasted 6 months. Longer-term studies of these combined interventions may be needed to estimate long-term effects for overweight and obese individuals.

The beneficial effects of combined exercise and low carbohydrate ketogenic diet may be associated with several possible mechanisms. Exercise training that includes aerobic and resistance training for overweight and obese individuals may enhance mitochondrial function including increased mitochondrial volume and protein turnover due to damaged protein degradation and new functional protein synthesis, skeletal muscle changes that include increased metabolic enzymes, ratio of capillaries to muscle fibers, insulin sensitivity, decreased catabolic mRNA expression, cardiac muscle changes including increased contractility and relaxation and decreased left ventricular stiffness, and conduit artery changes that include increased antioxidant expression, manganese, and decreased prooxidant expression that result in increased cardiovascular fitness [30]. Low carbohydrate ketogenic diets increase fat utilization due to enhanced metabolism due to carbohydrate to fat oxidation [31,32], higher HDL, and decreased triglycerides [33-35]. In addition, increased exercise intensity increases stimulation of muscle hormone sensitive lipase that leads to increased intra-muscular triglyceride hydrolysis [36,37].

A recommendation for a proper design regarding exercise and ketogenic diet is a randomized controlled trial (RCT) because this design helps reduce bias in the treatment 
effects by randomly allocating subjects to more than two groups including control and experimental groups. Additionally, the control groups need to use interventions of usual diet plus exercise and usual diet only without exercise. Seven of the selected studies, found based on the inclusion and exclusion criteria, were randomized controlled trials that used combined exercise and low carbohydrate ketogenic diet as experimental groups and usual diet plus exercise as the control groups. A previous study investigated the effects of a low carbohydrate ketogenic diet versus a control with a low fat diet to reduce fatness and hyperlipidemia, while the previous study did not include exercise interventions [12]. More high quality randomized controlled studies may be needed to produce stronger evidence of the combined effects of exercise and ketogenic diets.

\section{Conclusions}

Overweight and obese individuals who regularly engage in "yo-yo" dieting regimens and suffer from metabolic disorders need effective weight loss programs other than and continuously counting their caloric intake in every meal. This meta-analysis found effective interventions for these individuals. Overweight and obese individuals who participated in a combined exercise and low carbohydrate ketogenic diet intervention, had favorable effects of waist circumference and triglycerides post-intervention, but the body composition metrics, fasting glucose, and other lipid profiles were not statistically different post-intervention in the current meta-analysis. Additional studies are needed to confirm these findings due to the limited number of randomized controlled trials included, inconsistent control groups, and restricted measurements for each component including body composition metrics. Average exercise interventions in current meta-analysis were 9.2 weeks, moderate to vigorous intensity exercise, at 4 times per week, and $60 \mathrm{~min}$ per day of aerobic and resistance exercise with low carbohydrate ketogenic diet interventions. While more studies regarding longer term training in the combined exercise and low carbohydrate ketogenic diet interventions may be needed, the interventions for overweight and obese individuals in the current meta-analysis did not report any adverse side effects.

Supplementary Materials: The following are available online at https:/ / www.mdpi.com/1660-460 1/18/2/828/s1, Table S1: Assessments of the quality and risk of bias.

Author Contributions: Conceptualization, J.L and H.S.L.; methodology, J.L.; software, J.L.; validation, J.L.; formal analysis, J.L.; investigation, J.L.; resources, J.L.; data, J.L.; writing-original draft preparation, J.L. and H.S.L.; writing-review and editing, J.L.; visualization, J.L.; supervision, J.L.; project administration, J.L.; funding acquisition, J.L. All authors have read and agreed to the published version of the manuscript.

Funding: This research received no external funding.

Institutional Review Board Statement: Not applicable.

Informed Consent Statement: Not applicable.

Conflicts of Interest: The authors declare no conflict of interest.

\section{References}

1. WHO. Obesity and Overweight. Available online: https://www.who.int/news-room/fact-sheets/detail/obesity-andoverweight (accessed on 5 November 2020).

2. CDC, Center for Disease Control and Prevention. Overweight \& Obesity. Available online: https://www.cdc.gov/obesity/index. html (accessed on 5 November 2020).

3. Matsuda, M.; Shimomura, I. Increased oxidative stress in obesity: Implications for metabolic syndrome, diabetes, hypertension, dyslipidemia, atherosclerosis, and cancer. Obes. Res. Clin. Pract. 2013, 7, e330-e341. [CrossRef] [PubMed]

4. Katzmarzyk, P.T.; Church, T.S.; Janssen, I.; Ross, R.; Blair, S.N. Metabolic syndrome, obesity, and mortality: Impact of cardiorespiratory fitness. Diabetes Care 2005, 28, 391-397. [CrossRef] [PubMed]

5. Krauss, R.M.; Eckel, R.H.; Howard, B.; Appel, L.J.; Daniels, S.R.; Deckelbaum, R.J.; Erdman, J.W., Jr.; Kris-Etherton, P.; Goldberg, I.J.; Kotchen, T.A.; et al. AHA Dietary Guidelines: Revision 2000: A statement for healthcare professionals from the Nutrition Committee of the American Heart Association. Stroke 2000, 31, 2751-2766. [CrossRef] [PubMed] 
6. Garber, C.E.; Blissmer, B.; Deschenes, M.R.; Franklin, B.A.; Lamonte, M.J.; Lee, I.M.; Nieman, D.C.; Swain, D.P. American College of Sports Medicine position stand. Quantity and quality of exercise for developing and maintaining cardiorespiratory, musculoskeletal, and neuromotor fitness in apparently healthy adults: Guidance for prescribing exercise. Med. Sci. Sports Exerc. 2011, 43, 1334-1359. [CrossRef] [PubMed]

7. Aranceta, J.; Perez-Rodrigo, C. Recommended dietary reference intakes, nutritional goals and dietary guidelines for fat and fatty acids: A systematic review. Br. J. Nutr. 2012, 107 (Suppl. S2), S8-S22. [CrossRef]

8. Masood, W.; Annamaraju, P.; Uppaluri, K.R. Ketogenic Diet. In StatPearls; Treasure Island: Florida, FL, USA, 2020.

9. Cycling, W. National Task Force on the Prevention and Treatment of Obesity. JAMA 1994, 272, 1196-1202.

10. Gomez-Arbelaez, D.; Bellido, D.; Castro, A.I.; Ordonez-Mayan, L.; Carreira, J.; Galban, C.; Martinez-Olmos, M.A.; Crujeiras, A.B.; Sajoux, I.; Casanueva, F.F. Body Composition Changes After Very-Low-Calorie Ketogenic Diet in Obesity Evaluated by 3 Standardized Methods. J. Clin. Endocrinol. Metab. 2017, 102, 488-498. [CrossRef]

11. Garrow, J.S.; Summerbell, C.D. Meta-analysis: Effect of exercise, with or without dieting, on the body composition of overweight subjects. Eur. J. Clin. Nutr. 1995, 49, 1-10.

12. Yancy, W.S., Jr.; Olsen, M.K.; Guyton, J.R.; Bakst, R.P.; Westman, E.C. A low-carbohydrate, ketogenic diet versus a low-fat diet to treat obesity and hyperlipidemia: A randomized, controlled trial. Ann. Intern. Med. 2004, 140, 769-777. [CrossRef]

13. Noakes, M.; Foster, P.R.; Keogh, J.B.; James, A.P.; Mamo, J.C.; Clifton, P.M. Comparison of isocaloric very low carbohydrate/high saturated fat and high carbohydrate/low saturated fat diets on body composition and cardiovascular risk. Nutr. Metab. 2006, 3, 7. [CrossRef]

14. Liberati, A.; Altman, D.G.; Tetzlaff, J.; Mulrow, C.; Gotzsche, P.C.; Ioannidis, J.P.; Clarke, M.; Devereaux, P.J.; Kleijnen, J.; Moher, D. The PRISMA statement for reporting systematic reviews and meta-analyses of studies that evaluate health care interventions: Explanation and elaboration. Ann. Intern. Med. 2009, 151, W65-W94. [CrossRef] [PubMed]

15. Higgins, J.P.; Altman, D.G.; Gotzsche, P.C.; Juni, P.; Moher, D.; Oxman, A.D.; Savovic, J.; Schulz, K.F.; Weeks, L.; Sterne, J.A.; et al. The Cochrane Collaboration's tool for assessing risk of bias in randomised trials. BMJ 2011, 343, d5928. [CrossRef] [PubMed]

16. Wood, R.J.; Gregory, S.M.; Sawyer, J.; Milch, C.M.; Matthews, T.D.; Headley, S.A. Preservation of fat-free mass after two distinct weight loss diets with and without progressive resistance exercise. Metab. Syndr. Relat. Disord. 2012, 10, 167-174. [CrossRef] [PubMed]

17. Sun, S.; Kong, Z.; Shi, Q.; Hu, M.; Zhang, H.; Zhang, D.; Nie, J. Non-Energy-Restricted Low-Carbohydrate Diet Combined with Exercise Intervention Improved Cardiometabolic Health in Overweight Chinese Females. Nutrients 2019, 11, 3051. [CrossRef]

18. Freedland, S.J.; Howard, L.; Allen, J.; Smith, J.; Stout, J.; Aronson, W.; Inman, B.A.; Armstrong, A.J.; George, D.; Westman, E.; et al. A lifestyle intervention of weight loss via a low-carbohydrate diet plus walking to reduce metabolic disturbances caused by androgen deprivation therapy among prostate cancer patients: Carbohydrate and prostate study 1 (CAPS1) randomized controlled trial. Prostate Cancer Prostatic Dis. 2019, 22, 428-437. [CrossRef]

19. Perissiou, M.; Borkoles, E.; Kobayashi, K.; Polman, R. The Effect of an 8 Week Prescribed Exercise and Low-Carbohydrate Diet on Cardiorespiratory Fitness, Body Composition and Cardiometabolic Risk Factors in Obese Individuals: A Randomised Controlled Trial. Nutrients 2020, 12, 482. [CrossRef]

20. Jabekk, P.T.; Moe, I.A.; Meen, H.D.; Tomten, S.E.; Hostmark, A.T. Resistance training in overweight women on a ketogenic diet conserved lean body mass while reducing body fat. Nutr. Metab. 2010, 7, 17. [CrossRef]

21. Gregory, R.M.; Hamdan, H.; Torisky, D.M.; Akers, J.D. A low-carbohydrate Ketogenic diet combined with 6-weeks of Crossfit training improves body composition and performance. Int. J. Sports Exerc. Med. 2017, 3, 54. [CrossRef]

22. Poirier, P.; Despres, J.P. Waist circumference, visceral obesity, and cardiovascular risk. J. Cardiopulm. Rehabil. 2003, 23, 161-169. [CrossRef]

23. Perez-Martinez, P.; Alcala-Diaz, J.F.; Delgado-Lista, J.; Garcia-Rios, A.; Gomez-Delgado, F.; Marin-Hinojosa, C.; RodriguezCantalejo, F.; Delgado-Casado, N.; Perez-Caballero, A.I.; Fuentes-Jimenez, F.J.; et al. Metabolic phenotypes of obesity influence triglyceride and inflammation homoeostasis. Eur. J. Clin. Investig. 2014, 44, 1053-1064. [CrossRef]

24. Despres, J.P.; Arsenault, B.J.; Cote, M.; Cartier, A.; Lemieux, I. Abdominal obesity: The cholesterol of the 21st century? Can. J. Cardiol. 2008, 24 (Suppl. D), 7D-12D. [CrossRef]

25. Lee, J. Influences of Cardiovascular Fitness and Body Fatness on the Risk of Metabolic Syndrome: A Systematic Review and Meta-Analysis. Am. J. Health Promot. 2020, 34, 796-805. [CrossRef] [PubMed]

26. Yang, C.; Williams, J.M. Palladium-catalyzed cyanation of aryl bromides promoted by low-level organotin compounds. Org. Lett. 2004, 6, 2837-2840. [CrossRef] [PubMed]

27. Johnson, N.A.; Sachinwalla, T.; Walton, D.W.; Smith, K.; Armstrong, A.; Thompson, M.W.; George, J. Aerobic exercise training reduces hepatic and visceral lipids in obese individuals without weight loss. Hepatology 2009, 50, 1105-1112. [CrossRef] [PubMed]

28. Hall, K.D.; Chen, K.Y.; Guo, J.; Lam, Y.Y.; Leibel, R.L.; Mayer, L.E.; Reitman, M.L.; Rosenbaum, M.; Smith, S.R.; Walsh, B.T.; et al Energy expenditure and body composition changes after an isocaloric ketogenic diet in overweight and obese men. Am. J. Clin. Nutr. 2016, 104, 324-333. [CrossRef] [PubMed]

29. Guasch-Ferre, M.; Babio, N.; Martinez-Gonzalez, M.A.; Corella, D.; Ros, E.; Martin-Pelaez, S.; Estruch, R.; Aros, F.; Gomez-Gracia, E.; Fiol, M.; et al. Dietary fat intake and risk of cardiovascular disease and all-cause mortality in a population at high risk of cardiovascular disease. Am. J. Clin. Nutr. 2015, 102, 1563-1573. [CrossRef] 
30. Carbone, S.; Canada, J.M.; Billingsley, H.E.; Siddiqui, M.S.; Elagizi, A.; Lavie, C.J. Obesity paradox in cardiovascular disease: Where do we stand? Vasc. Health Risk Manag. 2019, 15, 89-100. [CrossRef]

31. Iwayama, K.; Kawabuchi, R.; Nabekura, Y.; Kurihara, R.; Park, I.; Kobayashi, M.; Ogata, H.; Kayaba, M.; Omi, N.; Satoh, M.; et al. Exercise before breakfast increases 24-h fat oxidation in female subjects. PLoS ONE 2017, 12, e0180472. [CrossRef]

32. Iwayama, K.; Kawabuchi, R.; Park, I.; Kurihara, R.; Kobayashi, M.; Hibi, M.; Oishi, S.; Yasunaga, K.; Ogata, H.; Nabekura, Y.; et al. Transient energy deficit induced by exercise increases 24-h fat oxidation in young trained men. J. Appl. Physiol. 2015, 118, 80-85. [CrossRef]

33. Wood, R.J.; Volek, J.S.; Davis, S.R.; Dell'Ova, C.; Fernandez, M.L. Effects of a carbohydrate-restricted diet on emerging plasma markers for cardiovascular disease. Nutr. Metab. 2006, 3, 19. [CrossRef]

34. Dashti, H.M.; Al-Zaid, N.S.; Mathew, T.C.; Al-Mousawi, M.; Talib, H.; Asfar, S.K.; Behbahani, A.I. Long term effects of ketogenic diet in obese subjects with high cholesterol level. Mol. Cell Biochem. 2006, 286, 1-9. [CrossRef] [PubMed]

35. Mensink, R.P.; Zock, P.L.; Kester, A.D.; Katan, M.B. Effects of dietary fatty acids and carbohydrates on the ratio of serum total to HDL cholesterol and on serum lipids and apolipoproteins: A meta-analysis of 60 controlled trials. Am. J. Clin. Nutr. 2003, 77, 1146-1155. [CrossRef] [PubMed]

36. Langfort, J.; Ploug, T.; Ihlemann, J.; Holm, C.; Galbo, H. Stimulation of hormone-sensitive lipase activity by contractions in rat skeletal muscle. Biochem. J. 2000, 351, 207-214. [CrossRef] [PubMed]

37. Storlien, L.H.; Jenkins, A.B.; Chisholm, D.J.; Pascoe, W.S.; Khouri, S.; Kraegen, E.W. Influence of dietary fat composition on development of insulin resistance in rats. Relationship to muscle triglyceride and omega-3 fatty acids in muscle phospholipid. Diabetes 1991, 40, 280-289. [CrossRef] [PubMed] 